\title{
Retrospective Evaluation of the Accuracy of Point of Care Versus Central Laboratory Sodium Measurements at a Supra Maximal Care Hospital
}

\section{Ramona C. Dolscheid-Pommerich ${ }^{*}$, Sarah Dolscheid ${ }^{2}$, Lars Eichhorn ${ }^{3}$, Birgit Stoffel-Wagner ${ }^{1}$, Ingo Graeff ${ }^{4}$}

\begin{abstract}
${ }^{1}$ Department of Clinical Chemistry and Clinical Pharmacology, University Hospital Bonn, Sigmund- Freud-Str. Bonn, Germany ${ }^{2}$ Department of Rehabilitation and Special Education, University of Cologne, Herbert-Lewin-Str. Köln, Germany ${ }^{3}$ Department of Anesthesiology and Intensive Care Medicine, University Hospital Bonn, Sigmund- Freud-Str. Bonn, Germany ${ }^{4}$ Center Clinician Scientist, Emergency Department, University Hospital Bonn, Sigmund- Freud-Str. Bonn, Germany
\end{abstract}

\begin{abstract}
:
Background: Sodium is most frequently requested by the emergency department (ED). It can be measured by direct or indirect ion-selective electrodes (ISE).

Aim: Comparison of sodium values obtained from POCT under proven concept conditions and Central Laboratory (CL) analyzers in an accredited laboratory in a large collective of patients. Do significant differences exist in sodium values obtained by direct/indirect ISE?

Methods: In 1941 patients, sodium values were established with POCT-blood gas analyzers in parallel with CL-analyzers. Differences between values were calculated. Clinical relevant outliers were evaluated according to reference and alarm ranges. Data were analyzed with correlation analysis, Bland-Altman plot and outlier evaluation.

Results: Average age was 55.7 years. Male-to-female ratio was 1149:792. Mean value of the absolute difference between POCT and CL sodium was $0.9 \mathrm{mmol} / 1$. POCT sodium mean value was $137.9 \mathrm{mmol} / \mathrm{l}$ (SD 4.7) and CL sodium mean value was $139.6 \mathrm{mmol} / \mathrm{l}$ (SD 4.2). POCT sodium and CL sodium correlated significantly $(\mathrm{r}=0.81, p<0.0001)$. Bland-Altman's mean difference of the measurement values was 1.6 (limits of agreement -3.8 to +7.1 ). 433 patients had sodium values outside of reference ranges. In 197, both values resulted in the same diagnosis. McNemar's test revealed significant differences $(p<0.0001) .9$ patients had values outside alarm ranges, in five of these cases, both values resulted in the same diagnosis.

Conclusion: We showed that in a large ED-collective of a supra maximal care hospital more than $85 \%$ of the patients had no clinically relevant discrepancies regarding their sodium values obtained with direct or indirect ISE.
\end{abstract}

\section{Keywords: Point-of Care Testing; Sodium; Ion-selective electrodes; Hypernatremia; Hyponatremia}

\section{Introduction}

Point of Care testing (POCT) has become increasingly important in recent years. In contrast to measurements performed in central laboratories, POCT requires lower sample volumes, less sample preparation procedures and no transport is necessary to obtain pre-analytics. In the hospital setting, POCT offers laboratory values right at the patient's bedside, enabling immediate diagnostic and/or therapeutic action. A major issue for debate is whether POCT should be performed by non-laboratory staff. Therefore, implementation of a statutory and successfully functioning POCT concept is mandatory in order to obtain reliable POCT results ${ }^{[1]}$. At the University Hospital Bonn, we have recently implemented and proven our POCT concept ${ }^{[1]}$.

Sodium is one of the parameters most frequently requested at the ED and it can be established with direct or indirect ion-selective electrodes or via flame photometry. While Point of Care (POCT) analyzers work with direct ion-selective electrodes, most of the laboratory analyzers in routine diagnostic work with indirect ion-selective electrodes. However, indirect ion-selective electrodes (ISE) are biased when there is a disproportion in the physiological protein/water ratio. This phenomenon is due to the ion exclusion effect, which
Received date: January 31, 2018 Accepted date: February 16, 2018 Published date: February 22, 2018

*Corresponding author: Ramona Dolscheid-Pommerich, MD, Sigmund-FreudStr. 25, 53127 Bonn, Germany, Tel: +49 228 287 12180/ Fax +49 228287 12159; E-mail: ramona.dolscheid-pommerich@ukbonn.de

Citation: Dolscheid-Pommerich, R.C., et al. Retrospective Evaluation of the Accuracy of Point of Care Versus Central Laboratory Sodium Measurements at a Supra Maximal Care Hospital. (2018) J Anesth Surg 5(1): 22-26.

Copyright: (C) 2018 Dolscheid-Pommerich, R.C. This is an Open access article distributed under the terms of Creative Commons Attribution 4.0 International License. 
has been known for a long time ${ }^{[2]}$. When using indirect ion-selective electrodes, a dilution of the sample is necessary and a fixed plasma water concentration of $93 \%$ is required. Therefore, hyper- or hypoproteinamia may lead to pseudohypo- or hypernatremia ${ }^{[3]}$. This potential bias is a problem not only in adult patients, but also in children and neonate ${ }^{[3,4]}$. Previous studies have revealed considerable differences in sodium values when comparing central laboratory sodium values with parallel-obtained POCT values $^{[5,6]}$. Therefore, the IFCC (International Federation of Clinical Chemistry and Laboratory Medicine) recommends direct ion-selective electrodes for sodium measurements in cases where plasma volumes are altered ${ }^{[7]}$. Thus, much effort has been spent to develop and improve laboratory methods in recent years and the statutory requirements have been continuously revised (guideline of the German Medical Association RiliBAEK) ${ }^{[8]}$.

\section{Importance of the study}

Laboratory methods are continuously improved and in recent years, legal requirements especially for POCT have been reinforced. Only a small number of studies are available dealing with the topic of potentially biased sodium values in daily routine diagnostics, often without providing a reliable transferability to a maximum care hospital[9]. The IFCC recommends direct ion-selective electrodes for sodium measurements in cases where plasma volumes are altered ${ }^{[7]}$. This presumes that a statutory and reliable POCT concept is provided for sodium measurements. While the IFCC strongly recommends direct ion-selective electrodes when plasma protein levels are altered we are confronted with the additional problem that very few total protein or albumin measurements are performed in blood values obtained at our emergency department.

\section{Aim of the study}

Aim of the present study was to assess the differences between sodium measurements obtained with POCT blood gas analyzers (Rapidlab 1265 $\mathrm{TM}$, Siemens Healthineers, Eschborn, Germany) and central laboratory measurements by Dimension VISTA1500 ${ }^{\mathrm{TM}}$ (Siemens Healthineers, Eschborn, Germany) in a large collective of patients presented at our emergency department. It was our intention to establish whether significant differences exist in sodium values obtained by direct or indirect ISE and if so, whether this influences the clinicians' decisions regarding diagnosis and therapies at the ED. For this purpose, we investigated how many clinically relevant discrepancies between sodium values actually occur.

\section{Materials and Methods}

\section{Setting}

The study was performed as a single-center retrospective observational study at the emergency department (ED) in collaboration with the central laboratory of the University Hospital Bonn (UKB), Germany. UKB is certified according to DIN EN ISO 9001:2008, while ED is certified as a level-1 trauma center. The central laboratory is accredited according to DIN EN ISO 15189:2014. Currently, there are approximately 2600 trained POCT users at UKB where all disciplines are represented with 1250 beds in total. Blood sampling for the central laboratory and for POCT measurement were performed simultaneously.
Samples for the central laboratory were transferred immediately via a rapid pneumatic transport system. Details on our POCT concept have been published previously and during the whole evaluation period, the entire setting, including workflow, number of staff, POCT training etc. both at ED and the central laboratory remained unchanged ${ }^{[]}$. The external and internal quality control requirements for POCT as well as central laboratory measurements conform strictly to legal requirements.

\section{Central laboratory measurements}

Sodium values at the central laboratory were obtained with the analyzer Dimension VISTA1500TM (Siemens Healthineers, Eschborn, Germany) via indirect ISE. Examination material consisted of venous serum. In all samples, analysis was performed immediately after arrival at the central laboratory and centrifugation for ten minutes.

\section{POCT measurements}

At ED, sodium values were obtained with Rapidlap $^{\mathrm{TM}} 1265$ (Siemens Healthineers, Eschborn, Germany) analyzer via direct ISE. Examination material for blood gas analysis was heparinized venous whole blood. Analysis was performed immediately and the blood samples for POCT and the blood samples for central laboratory analysis were obtained simultaneously.

\section{Collective}

We analyzed data on POCT sodium measurements and CL sodium measurements from 1941 patients who attended our emergency department during a period of one year (January 2016 to December 2016, Raw Data see S1.). Next, we analyzed/ calculated the following values:

1. Absolute values of sodium were collated with the differences between POCT sodium and CL sodium measurements.

2. Outlier evaluation regarding reference ranges for sodium; reference ranges $135-145 \mathrm{mmol} / \mathrm{l}$

3. Outlier evaluation regarding alarm ranges for sodium; alarm ranges: $<120 \mathrm{mmol} / \mathrm{l}$ and $>160 \mathrm{mmol} / \mathrm{l}$

\section{Statistics}

Data were statistically analyzed using Microsoft Excel, version 2007 and MedCalc $\AA$, version 11.0.0.0. We considered $p<0.05$ as statistically significant. Descriptive parameters (age and absolute difference of sodium) are reported with mean value $\pm \mathrm{SD}$, minimum and maximum and male-to-female ratio was described. Next, sodium values obtained with POCT devices and with central laboratory devices were described with mean value $\pm \mathrm{SD}$ (standard deviation). Correlation analyses and t-tests to compare the sodium measurement results were performed. We calculated the difference between POCT sodium and CL sodium (CL sodium - POCT sodium) for each patient and plotted it against their mean value ((CL sodium + POCT sodium)/2) to summarize data in a Bland-Altman plot. We described the limits of agreement of the Bland-Altman plot (i.e. the interval within which $95 \%$ of differences between measurements by the two methods are expected to lie). Finally, we analyzed outliers regarding the reference ranges for sodium (135-145 mmol/l) and alarm ranges $(<120 ;>160 \mathrm{mmol} / \mathrm{l})$. 
Citation: Dolscheid-Pommerich, R.C., et al. Retrospective Evaluation of the Accuracy of Point of Care Versus Central Laboratory Sodium Measurements at a Supra Maximal Care Hospital. (2018) J Anesth Surg 5(1): 22- 26.

\section{Ethics}

This was a single-center retrospective observational study. Therefore, according to paragraph 6 of the German Data Protection Act, the physician may use existing patient data for retrospective analyses without explicitly asking for the consent of patients. Furthermore, all collected data were fully anonymized before analysis. The local ethics commission (directorship Prof. Racké) states that a retrospective analysis of data obtained during routine treatment and diagnosis does not require consultation by the ethics commission pursuant to paragraph 15 of the medical professional code.

\section{Results}

We analyzed data from 1941 patients with an average age of 55.7 years (range 5 - 102 years). Male-to-female ratio was 1149 to 792 (59.2\% - 40.8\% male- female patients). Mean value of the absolute difference between POCT sodium and CL sodium was $0.9 \mathrm{mmol} / \mathrm{l}$ with a range from 0 to 21 . Table 1 shows absolute differences for the obtained sodium values. For POCT sodium, the mean value was $137.9 \mathrm{mmol} / \mathrm{l}$ (SD 4.7) and for CL sodium, the mean value was $139.6 \mathrm{mmol} / \mathrm{l}$ (SD 4.2 $\mathrm{mmol} / \mathrm{l})$. Analysis of the correlation between POCT sodium and CL sodium revealed a significant correlation with $r=0.81, p$ $<0.0001$. On average, CL sodium values tended to be higher than POCT sodium values $(t(1941) p<0.0001)$. Scatter diagram with regression line is shown in Figure 1. Investigation of the mean difference between measurement values using Bland-Altman plot revealed a value of $1.6 \mathrm{mmol} / \mathrm{l}$. Bland-Altman plot is shown in Figure 2 with limits of agreement ranging from -3.8 to $+7.1 \mathrm{mmol} / \mathrm{l}$. Outlier evaluation regarding reference ranges for sodium revealed $n=433$, while $n=9$ where outliers regarding alarm ranges for sodium. All outliers were systematically analyzed (see below) with cross-tables, sensitivity, specificity and McNemar tests.

Table 1: Absolute difference between POCT sodium and central laboratory sodium measurements.

\begin{tabular}{|c|c|}
\hline Absolute difference $(\mathrm{mmol} / \mathrm{l})$ & Samples \\
\hline$(\%)$ & $1683(86.7)$ \\
\hline$<4$ & $126(6.5)$ \\
\hline 5 & $45(2.3)$ \\
\hline 6 & $34(1.8)$ \\
\hline 7 & $16(0.8)$ \\
\hline 8 & $13(0.7)$ \\
\hline 9 & $4(0.2)$ \\
\hline 10 & $7(0.4)$ \\
\hline 11 & $2(0.1)$ \\
\hline 12 & $5(0.3)$ \\
\hline 13 & $1(0.1)$ \\
\hline 15 & $2(0.1)$ \\
\hline 16 & $2(0.1)$ \\
\hline 17 & $1(0.1)$ \\
\hline 21 & \\
\hline
\end{tabular}

Table 1 shows the absolute differences in $\mathrm{mmol} / \mathrm{l}$ between POCT sodium and central laboratory sodium measurements (deviations in percentage distribution are due to rounding).
Figure 1: Correlation between sodium values (POCT vs. central laboratory).

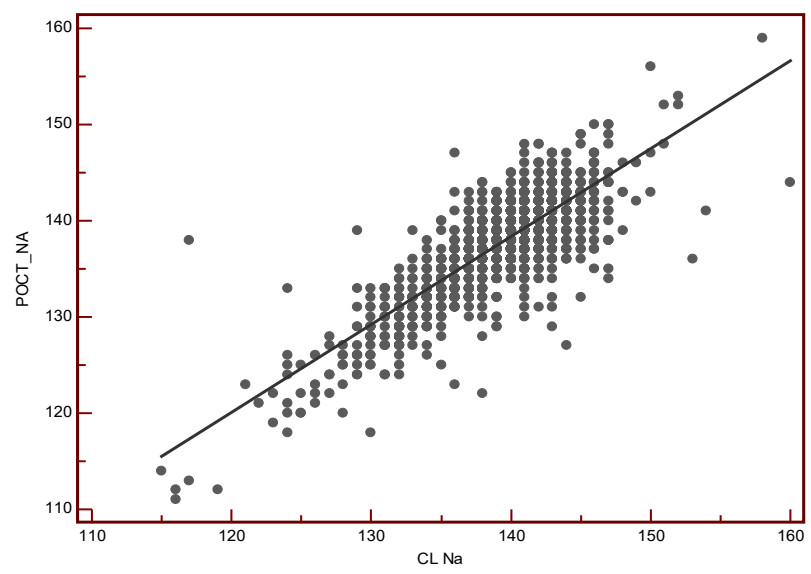

Figure 1 shows the correlation between sodium parameters obtained with POCT (POCT_Na) and a central laboratory analyzer in $\mathrm{mmol} / \mathrm{l}$ $\left(\mathrm{CL} \_\mathrm{Na}\right)$.

Figure 2: Bland-Altman plot for the difference of sodium measurements.

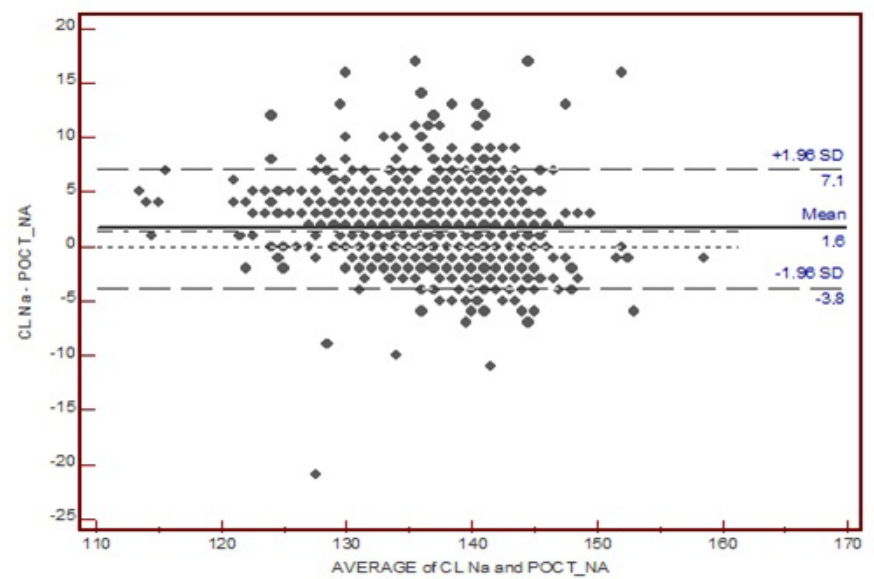

Figure 2 shows the difference between POCT sodium (POCT_Na) and sodium measured with central laboratory analyzers (CL-Na) calculated for each patient and plotted against the mean value of both measurements ((CL-Na + POCT-Na)/2).

Bold line shows the mean difference of the measurement values (1.6). The interrupted outer lines represent the limits of agreement within which $95 \%$ of differences between measurements by the two methods are expected to lie $(-3.8$ and 7.1$)$.

\section{Outlier evaluation regarding sodium reference ranges}

We evaluated the differences between both measurement methods regarding the sodium reference ranges (reference range for sodium: $(135-145 \mathrm{mmol} / \mathrm{l})$. In $\mathrm{n}=433$ patients $(22.3 \%)$, values outside the reference ranges for sodium were found. In 197 of these patients, both values (POCT and CL) resulted in the same diagnosis (hyper-or hyponatremia). Male to female ratio was 253 to 180 (58.4\% - 41.6\% male - female patients) and average age was 62.8 years (range $17-101$ years). Mean sodium values were $133.5 \mathrm{mmol} / \mathrm{l}$ (SD $6.6 \mathrm{mmol} / 1$, min $120 \mathrm{mmol} / \mathrm{l}$, max. $159 \mathrm{mmol} / \mathrm{l}$ ) for POCT sodium and $136.5 \mathrm{mmol} / \mathrm{l}$ (SD 6.3 $\mathrm{mmol} / \mathrm{l}$, min $121 \mathrm{mmol} / 1$, max. $160 \mathrm{mmol} / \mathrm{l}$ ) for CL sodium. Table 2 shows the cross-table in this outlier collective regarding sodium reference ranges. The McNemar test revealed significant 
differences $(p<0.0001)$ when comparing both values (POCT sodium vs. CL sodium) and CL sodium resulted significantly more often in a diagnosis of hyper-or hyponatremia.

Table 2: Cross-tables outlier sodium reference ranges

\begin{tabular}{|l|l|l|l|}
\hline \multicolumn{3}{|l|}{ Outlier sodium reference ranges } \\
\hline & CL-Na & CL-Na & sens. 52.4\% \\
\hline & in (\%) & out (\%) & spec. 96.4\% \\
\hline POCT-Na in (\%) & $197(62.5)$ & $57(0.05)$ & 254 \\
\hline POCT-Na out (\%) & $179(37.5)$ & $1508(99.95)$ & 1687 \\
\hline & $376(100)$ & $1565(100)$ & 1941 \\
\hline
\end{tabular}

Table 2 shows the cross-table with sensitivity (sens.) and specificity (spec.) for POCT measurement in patients with sodium levels outside reference ranges. "In" indicates that patients are classified as outside of reference ranges by the respective method (POCT, CL), whereas "out" indicates that patients have sodium values within the reference ranges according to the respective method.

\section{Outlier evaluation regarding sodium alarm ranges}

In $\mathrm{n}=9$ patients $(0.5 \%)$, values outside the clinically important cut-off alarm ranges for sodium occurred (alarm ranges for sodium: $\left.(<120 ;>160 \mathrm{mmol} / \mathrm{l})^{[10]}\right)$. In five of these cases, both values resulted in the same diagnosis (hyponatremia). Male to female ratio was six to three $(66.7 \%-33.3 \%$ male - female patients) and average age was 59.7 (range $43-81$ years), while mean sodium values were $117.2 \mathrm{mmol} / 1$ (SD $8.3 \mathrm{mmol} / 1$, min $111 \mathrm{mmol} / \mathrm{l}$, max. $138 \mathrm{mmol} / \mathrm{l})$ ) for POCT sodium and 119.7 $\mathrm{mmol} / \mathrm{l}(\mathrm{SD} 5.0 \mathrm{mmol} / \mathrm{l}, \min 115 \mathrm{mmol} / \mathrm{l}$, max. $130 \mathrm{mmol} / \mathrm{l})$ ) for CL sodium. Table 3 shows the cross-table in this outlier collective regarding sodium alarm ranges. McNemar test revealed no significant differences when comparing both measurement methods regarding the critically alarm ranges $(p=0.625)$.

Table 3: Cross-tables outlier sodium alarm ranges

\begin{tabular}{|l|l|l|l|}
\hline Outlier alarm ranges & CL-Na & CL-Na & sens. $62.5 \%$ \\
\hline & in (\%) & out (\%) & spec. $99.6 \%$ \\
\hline POCT-Na in (\%) & $5(62.5)$ & $1(0.05)$ & 6 \\
\hline POCT-Na out (\%) & $3(37.5)$ & $1932(99.95)$ & 1936 \\
\hline & $8(100)$ & $1933(100)$ & 1941 \\
\hline
\end{tabular}

Table 3 shows the cross-table with sensitivity (sens.) and specificity (spec.) for POCT measurement in patients with sodium outside alarm ranges. "In" indicates that patients are classified as within the alarm range by the respective method (POCT, CL), whereas "out" indicates that patients have sodium values within the reference ranges according to the respective method.

\section{Discussion}

In our collective, $>85 \%$ of the patients had no clinically relevant discrepancies regarding their sodium values when comparing POCT blood gas analysis with results obtained from the central laboratory analyzer. In our large collective, we analyzed important clinical subgroups according to the reference ranges and alarm ranges for sodium. As samples for POCT as well as central laboratory analysis were obtained simultaneously and since both examination materials consisted of venous blood, excellent comparability was achieved. Previous studies revealed significant biases when comparing venous plasma analyzed by indirect ISE with capillary blood analyzed by direct ISE ${ }^{[1]}$. We recommend a two-step procedure: First, a highly recommended validation of the present POCT concept followed by an evaluation of sodium levels in individual settings to allow for assay specificities and individual patient collectives. Our analysis was performed in the setting of an accredited central laboratory, an accredited ED and a POCT concept in line with statutory requirements ${ }^{[1]}$. A high number of patients enabled analysis of wide ranges of sodium values including hypo-or hypernatremia. Studies have shown that even when the same POCT analyzer is used, different values may be obtained if there was no instruction in pre-analytic handling, which constitutes a major part of the POCT user training ${ }^{[12]}$. Although we showed that $>85 \%$ of the patients had no clinically relevant discrepancies regarding sodium values, outlier evaluation regarding important clinical cut-offs is mandatory. Several previous studies have reported discrepancies, but failed to evaluate whether these were clinically important ${ }^{[13]}$. Clinically important are any discrepancies regarding the diagnosis of hyper-or hyponatremia (including alarm value). However, previous studies with older analyzers are not transferrable to actual conditions ${ }^{[14]}$. Recently, Gavala et al. reported that a POCT analyzer significantly underestimates, inter alia, sodium when comparing results with central laboratory levels ${ }^{[9]}$. This study shows that - although IFCC recommends indirect ISE sodium measurements - the difficulties lie within a reliably working and statutory POCT concept, which is essential to obtain reliable POCT results. Therefore, in cases where clinically important discrepancies occur, we recommend to contact the laboratory to evaluate whether the pre-analytical conditions in POCT might have been biased or whether the patient's protein-water ratio has been affected, which can bias indirect ISE measurement. For pre-analytical problems in POCT sodium measurement, a specific troubleshooting procedure, e.g. under the supervision of the POCT coordination, is recommended. To assess whether the protein-water ratio is affected, albumin or total protein should be measured and if affected, POCT values should be preferred. In four patients, differences regarding the important cut-off for alarm ranges for sodium occurred. These four patients suffered from diseases accompanied by dehydration. Despite the fact that our POCT concept had been recently verified and laboratory techniques are steadily improving, we detected cases with major discrepancies. Therefore, we propose that clinicians are made aware of potential biases in sodium measurements and if these occur, that contact is made with the central laboratory to decide on the preferable measurement method. As POCT measurement techniques as well as those provided by central laboratory analyzers are continuously improved, future studies are needed to evaluate analyzers and methods.

\section{Limitations of the study}

We are confronted with the phenomenon that albumin or protein values are not requested upon first blood sampling at the ED. We evaluated sodium values from our ED because sodium for POCT as well as CL analysis is measured in venous blood samples. For CL analysis, serum is used, whereas for POCT, heparinized samples are employed. We referred to clinically important cut-offs and did not focus on laboratory errors of measurements. In our study, we performed a comparison 
Citation: Dolscheid-Pommerich, R.C., et al. Retrospective Evaluation of the Accuracy of Point of Care Versus Central Laboratory Sodium Measurements at a Supra Maximal Care Hospital. (2018) J Anesth Surg 5(1): 22- 26.

between currently used CL analyzers and POCT analyzer.

\section{Conclusions}

Overall, several of the patients in our study collective had no clinically relevant discrepancies regarding their sodium values. Nevertheless, outlier evaluation under a working POCT concept is mandatory in individual clinical settings to improve the quality of diagnosing and treating hypo-or hypernatremia as in individual cases, significant differences in sodium values obtained with direct and indirect ISE may remain. This can have an important impact on the clinician's choice of diagnosis and therapy. Therefore, training of clinicians remains important and we recommend detailed information exchange between laboratory physicians and clinicians in cases where significant discrepancies occur.

\section{Acknowledgement: None}

Supporting Information: S1 file. Raw data. Raw data including POCT sodium values, Central laboratory sodium values, absolute difference, sex and age.

\section{References}

1. Dolscheid-Pommerich, R.C., Dolscheid, S., Grigutsch, D., et al. Comparability of Point-of-Care versus Central Laboratory Hemoglobin Determination in Emergency Patients at a Supra-Maximal Care Hospital. (2016) PLoS One 11(11): e0166521.

PubMed |Crossref| Others

2. Ladenson, J.H., Apple, F.S., Aguanno, J.J., et al. Sodium measurements in multiple myeloma: two techniques compared. (1982) Clin Chem 28(12): 2383-2386.

PubMed | Crossref| Others

3. King, R.I., Mackay, R.J., Florkowski, C.M., et al. Electrolytes in sick neonates - which sodium is the right answer? (2013) Arch Dis Child Fetal Neonatal Ed 98(1): F74-F76.

PubMed |Crossref $\mid$ Others

4. Chhapola, V., Kanwal, S.K., Sharma, R., et al. A comparative study on reliability of point of care sodium and potassium estimation in a pediatric intensive care unit. (2013) Indian J Pediatr 80(9): 731-735.

PubMed | Crossref | Others

5. Chacko, B., Peter, J.V., Patole, S., et al. Electrolytes assessed by point-of-care testing - Are the values comparable with results obtained from the central laboratory? (2011) Indian J Crit Care Med 15(1): 2429.

PubMed | Crossref | Others

6. Jain, A., Subhan, I., Joshi, M. Comparison of the point-of-care blood gas analyzer versus the laboratory auto-analyzer for the measurement of electrolytes. (2009) Int J Emerg Med 2(2): 117-120.

PubMed | Crossref| Others

7. Burnett, R.W., Covington, A.K., Fogh-Andersen, N., et al. Recommendations for measurement of and conventions for reporting sodium and potassium by ion-selective electrodes in undiluted serum, plasma or whole blood. International Federation of Clinical Chemistry and Laboratory Medicine (IFCC). IFCC Scientific Division Working Group on Selective Electrodes. (2000) Clin Chem Lab Med 38(10): 1065-1071. PubMed | Crossref | Others

8. Richtlinie der Bundesärztekammer zur Qualitätssicherung laboratoriumsmedizinischer Untersuchungen - Rili-BÄK. (2014) Deutsches Ärzteblatt 111(38).

PubMed | Crossref $\mid$ Others

9. Gavala, A., Myrianthefs, P. Comparison of point-of-care versus cen- tral laboratory measurement of hematocrit, hemoglobin, and electrolyte concentrations. (2017) Heart Lung 46(4): 246-250.

PubMed | Crossref| Others

10. Kritische Laborergebnisse, die dem behandelnden Arzt sofort mitgeteilt wer-den müssen. (2017) eJIFCC 14(1)

PubMed | Crossref| Others

11. Loughrey, C.M., Hanna, E.V., McDonnell, M., et al. Sodium measurement: effects of differing sampling and analytical methods. (2006) Ann Clin Biochem 43(Pt 6): 488-493.

PubMed | Crossref | Others

12. Auvet, A., Espitalier, F., Grammatico-Guillon, L., et al. Preanalytical conditions of point-of-care testing in the intensive care unit are decisive for analysis reliability. (2016) Ann Intensive Care 6: 57.

PubMed | Crossref | Others

13. Weld, B.A., Morgan, T.J., Presneill, J.J., et al. Plasma sodium measurements by direct ion selective methods in laboratory and point of care may not be clinically interchangeable. (2016) J Clin Monit Comput 31(5): 1103-1109.

PubMed | Crossref | Others

14. Jain, A., Subhan, I., Joshi, M. Comparison of the point-of-care blood gas analyzer versus the laboratory auto-analyzer for the measurement of electrolytes. (2009) Int J Emerg Med 2(2): 117-120.

PubMed | Crossref | Others
Submit your manuscript to Ommega Publishers and we will help you at every step:

- We accept pre-submission inquiries

- Our selector tool helps you to find the most relevant journal

-We provide round the clock customer support

- Convenient online submission

- Thorough peer review

- Inclusion in all major indexing services

- Maximum visibility for your research

Submit your manuscript at

https://www.ommegaonline.org/submit-manuscript 